\title{
Multi-method genome- and epigenome- wide studies of inflammatory protein levels in healthy older adults
}

Robert F. Hillary ${ }^{1}$, Daniel Trejo-Banos², Athanasios Kousathanas², Daniel L. McCartney' ${ }^{1}$ Sarah E. Harris ${ }^{3,4}$, Anna J. Stevenson ${ }^{1}$, Marion Patxot ${ }^{2}$, Sven Erik Ojavee ${ }^{2}$, Qian Zhang ${ }^{5}$, David C. Liewald ${ }^{3}$, Craig W. Ritchie ${ }^{6}$, Kathryn L. Evans ${ }^{1}$, Elliot M. Tucker-Drob ${ }^{7,8}$, Naomi R. Wray ${ }^{5}$, Allan F. McRae ${ }^{5}$, Peter M. Visscher ${ }^{5}$, lan J. Deary ${ }^{3,4}$, Matthew R. Robinson ${ }^{9 *}$ and Riccardo E. Marioni ${ }^{*}$ (D)

\begin{abstract}
Background: The molecular factors which control circulating levels of inflammatory proteins are not well understood. Furthermore, association studies between molecular probes and human traits are often performed by linear modelbased methods which may fail to account for complex structure and interrelationships within molecular datasets.

Methods: In this study, we perform genome- and epigenome-wide association studies (GWAS/EWAS) on the levels of 70 plasma-derived inflammatory protein biomarkers in healthy older adults (Lothian Birth Cohort 1936; $n=876$; Olink ${ }^{\otimes}$ inflammation panel). We employ a Bayesian framework (BayesR+) which can account for issues pertaining to data structure and unknown confounding variables (with sensitivity analyses using ordinary least squares- (OLS) and mixed model-based approaches).

Results: We identified 13 SNPs associated with 13 proteins ( $n=1$ SNP each) concordant across OLS and Bayesian methods. We identified $3 \mathrm{CpG}$ sites spread across 3 proteins ( $n=1 \mathrm{CpG}$ each) that were concordant across OLS, mixedmodel and Bayesian analyses. Tagged genetic variants accounted for up to $45 \%$ of variance in protein levels (for MCP2, $36 \%$ of variance alone attributable to 1 polymorphism). Methylation data accounted for up to $46 \%$ of variation in protein levels (for CXCL10). Up to 66\% of variation in protein levels (for VEGFA) was explained using genetic and epigenetic data combined. We demonstrated putative causal relationships between CD6 and IL18R1 with inflammatory bowel disease and between IL12B and Crohn's disease.
\end{abstract}

Conclusions: Our data may aid understanding of the molecular regulation of the circulating inflammatory proteome as well as causal relationships between inflammatory mediators and disease.

\footnotetext{
* Correspondence: matthew.robinson@ist.ac.at; riccardo.marioni@ed.ac.uk ${ }^{9}$ Institute of Science and Technology Austria, 3400 Klosterneuburg, Austria ${ }^{1}$ Centre for Genomic and Experimental Medicine, Institute of Genetics and Molecular Medicine, University of Edinburgh, Edinburgh EH4 2XU, UK Full list of author information is available at the end of the article
}

(c) The Author(s). 2020 Open Access This article is licensed under a Creative Commons Attribution 4.0 International License, which permits use, sharing, adaptation, distribution and reproduction in any medium or format, as long as you give appropriate credit to the original author(s) and the source, provide a link to the Creative Commons licence, and indicate if changes were made. The images or other third party material in this article are included in the article's Creative Commons licence, unless indicated otherwise in a credit line to the material. If material is not included in the article's Creative Commons licence and your intended use is not permitted by statutory regulation or exceeds the permitted use, you will need to obtain permission directly from the copyright holder. To view a copy of this licence, visit http://creativecommons.org/licenses/by/4.0/ The Creative Commons Public Domain Dedication waiver (http://creativecommons.org/publicdomain/zero/1.0/) applies to the data made available in this article, unless otherwise stated in a credit line to the data. 


\section{Background}

Inflammation represents a concerted cascade of molecular and cellular events to combat infectious pathogens and endogenous insults. Inflammatory proteins are key mediators of defence and repair responses, and tight spatiotemporal regulation of their plasma concentrations permits effective immune activation and resolution [1]. Whereas acute inflammatory states may prompt severe illness and death, absence of resolution precipitates transition from acute to deleterious chronic inflammatory states [2]. Chronic inflammation facilitates the pathogenesis of various disease states, including diabetes, heart disease, stroke and allergic conditions [3]. Furthermore, inflammatory lesions in brain tissue are often associated with, and may contribute to, neurodegeneration and cognitive decline [4]. Globally, $60 \%$ of individuals will die as a consequence of a chronic inflammationassociated disease state [5]. Therefore, identifying biological factors which govern inter-individual variation in circulating inflammatory protein levels may allow for better prediction of individual disease risk and prognosis, and inform disease biology.

To date, a number of studies have aimed to characterise genetic factors associated with the levels of single inflammatory proteins or a small number of such proteins, including C-reactive protein, fibrinogen and interleukin6 [6-25]. These genetic factors are also known as protein quantitative trait loci or pQTLs. Additionally, studies have examined the genetic architecture of panels of proteins, including inflammatory mediators, and have investigated co-regulatory pathways and associations with disease states [26-35]. Instead of using imputed genotype data, Höglund et al. used whole genome sequencing data to carry out genome-wide association studies (GWAS) on the levels of 72 inflammatory proteins. This led to the identification of 18 novel loci that were not identified using genotyped or imputed SNPs [36]. A number of studies have also carried out epigenome-wide association studies (EWAS) on the levels of a small set of inflammatory proteins, including C-reactive protein, interleukins-( $1 \beta, 4,6,9$ and 10), interferon-gamma, transforming growth factor-beta and tumour necrosis factor [37-42]. Zaghlool et al. performed an EWAS of 1123 proteins, which pointed towards networks of chronic low-grade inflammatory biomarkers $(n=944$ individuals) [43]. In an integrative approach, Ahsan et al. aimed to identify genetic and epigenetic markers associated with protein biomarkers including inflammatory mediators ( $n \leq 1033$ individuals) [44]. No study has modelled GWAS and EWAS both as stand-alone association studies and in a combined analysis in the context of proteomic data. This would allow for the identification of genetic and epigenetic correlates of inflammatory protein levels and for the estimation of variance in protein levels explained by genetic and epigenetic data, considered in isolation but also conditioned on one another to reflect reciprocal influences of these molecular data types. Here, we triangulate results from multiple statistical approaches to provide a robust set of genetic and epigenetic correlates of inflammatory protein levels.

Notably, most studies examining the molecular architecture of human traits have relied on linear modelbased methods which examine marker or probe effects marginally $[45,46]$. A number of issues may arise when using linear regression-based methods and if these are not addressed in the study design, it may lead to model overfitting and biased estimation of effect sizes. These potential issues include correlation structure within molecular datasets, data structure (i.e. cellular heterogeneity, batch effects) and omitted variable bias [47]. Several approaches have been proposed to address these issues [47-53] and these encompass strategies which permit the joint and conditional estimation of effect sizes whilst accounting for correlations among markers and confounding variables. Here, we consider a Bayesian penalised regression framework termed BayesR+ which was developed to assess genetic and epigenetic architectures of complex traits [54]. In BayesR+, marker effects (SNP or CpG site) can be estimated jointly whilst controlling for data structure and correlations among molecular markers of different types. Indeed, this method permits the estimation of variance explained in the trait by all methylation probes or genetic markers, either separately or together. BayesR+ has been shown to outperform single-probe linear regression and penalised regression approaches, such as ridge and LASSO, in relation to the correlation of estimated effects with true simulated values as well as mean squared errors between true and estimated coefficients for single-probe regression. Additionally, BayesR + shows a higher correlation between estimated effects for variance explained by genetic and epigenetic markers in phenotypic traits and true simulated values when compared to a mixed model strategy in both sparse and non-sparse marker settings [54].

In the present study, we use the BayesR+ method (and sensitivity analyses using ordinary least squares (OLS) [55, 56] and mixed model methods [57]) to examine both the genetic and epigenetic architectures of 70 blood inflammatory proteins in 876 relatively healthy older adults from the Lothian Birth Cohort 1936 study (mean age $69.8 \pm 0.8$ years; levels adjusted for age, sex, population structure and array plate). Hereinafter, we refer to the adjusted inflammatory protein levels as protein levels. These proteins are present on the Olink ${ }^{\circ}$ inflammation panel and comprise a mixture of proteins with defined functions pertinent to human inflammatory pathways as well as putative roles in inflammation- 
related disease states. We use priors guided by results from previous genome-wide and epigenome-wide studies $[54,58]$ for the expected variance explained in circulating protein levels by genetic and epigenetic factors. Applying a stringent approach, we only consider markers or probes that were identified across all methods employed as being associated with a given protein (concordantly identified) and integrate multiple levels of 'omics' data to investigate mechanisms by which genetic variants may influence protein levels. Finally, we use our GWAS summary data to test for putatively causal relationships between inflammatory protein biomarkers and neurological or inflammatory disease states. Thus, this paper has two major aims. The first aim is to provide robust and novel estimates for the contribution of genetic and epigenetic factors towards inter-individual variation in circulating inflammatory protein concentrations. The relationships between genetic and epigenetic factors with inflammatory proteins levels are modelled both alone and together. The second aim is to provide the first use of multiple statistical methods in performing genomewide and epigenome-wide association studies of human proteomic data.

\section{Methods}

\section{The Lothian Birth Cohort 1936}

The Lothian Birth Cohort 1936 (LBC1936) study is a longitudinal study of ageing. Cohort members were all born in 1936 and most took part in the Scottish Mental Survey 1947 at age 11 years. Participants who were living mostly within the Edinburgh area were re-contacted approximately 60 years later $(n=1091$, recruited at mean age 70 years). Recruitment and testing of the LBC1936 cohort have been described previously [59, 60].

\section{Protein measurements in the Lothian Birth Cohort 1936}

Plasma was extracted from 1047 blood samples and collected in lithium heparin tubes at mean age $69.8 \pm 0.8$ years. Following quality control, 1017 samples remained. Plasma samples were analysed using a 92-plex proximity extension assay (Olink ${ }^{\bullet}$ Bioscience, Uppsala Sweden). One protein from the panel, BDNF, failed quality control and was removed from the study. For a further 21 proteins, over $40 \%$ of samples fell below the lowest limit of detection. These proteins were removed from analyses leaving a final set of 70 proteins. The proteins assayed comprise the Olink ${ }^{\diamond}$ inflammatory biomarker panel. Briefly, $1 \mu \mathrm{L}$ of sample was incubated in the presence of proximity antibody pairs linked to DNA reporter molecules. Upon appropriate antigen-antibody recognition, the DNA tails form an amplicon by proximity extension which is quantified by real-time PCR. Data preprocessing was performed by Olink ${ }^{\odot}$ using NPX Manager software. Protein levels were transformed by rank-based inverse normalisation and regressed onto age, sex, four genetic principal components of ancestry and array plate. Standardised residuals from these regression models were brought forward for all genetic-protein and epigenetic-protein analyses. Pre-adjusted protein level distributions are presented in Additional file 1. Associations between pre-adjusted protein levels and biological as well as technical covariates are detailed in Additional file 2: Table S1.

\section{Genome-wide association studies}

LBC1936 DNA samples were genotyped at the Edinburgh Clinical Research Facility using the Illumina 610Quadv1 array $(n=1005$; mean age $69.6 \pm 0.8$ years; San Diego). Quality control procedures for genetic data are detailed in Additional file 3.

BayesR+ is a software implemented in $\mathrm{C}++$ for performing Bayesian penalised regression on complex traits [54]. The joint and conditional effects of typed SNPs ( $n=521,523$ variants) on transformed protein levels were examined. The prior distribution is specified as a mixture of Gaussian distributions, corresponding to effect sizes of different magnitude, and a discrete spike at zero which enables the omission of probes and markers with negligible effect on the phenotype. Informed by data from our previous pQTL study [58], mixture variances for genetic data were set to 0.01 and 0.1 for the standalone BayesR+ GWAS. In the combined analysis with epigenetic data, owing to the need for the same number of mixture variances for genetic and epigenetic data in the BayesR+ software, mixture variances were set to $0.01,0.1$ and 0.2 . Input data were scaled to mean zero and unit variance, and adjusted for age and sex. To obtain estimates of effect sizes, Gibbs sampling was used to sample over the posterior distribution conditional on the input data. The Gibbs algorithm consisted of 10000 samples and 5000 samples of burn-in after which a thinning of 5 samples was utilised to reduce autocorrelation. Genetic markers which exhibited a posterior inclusion probability of $\geq 95 \%$ were deemed to be significant.

Details for the OLS regression model approach are outlined in Additional file 3. In the linear method, markers which surpassed a Bonferroni-corrected conditional significance threshold of $7.14 \times 10^{-10}$ (= genomewide significance $5.0 \times 10^{-8} / 70$ phenotypes) were considered. The genome-wide significance level of $5.0 \times 10^{-8}$ was selected as per convention in GWAS studies.

\section{Epigenome-wide association studies}

DNA from whole blood was assessed using the Infinium $450 \mathrm{~K}$ methylation array at the Edinburgh Clinical Research Facility ( $n=876$; mean age $69.8 \pm 0.8$ years). Quality control procedures for methylation data are detailed in Additional file 3. 
Using BayesR+, prior mixture variances for methylation data ( $n=459,309$ CpG sites) were set to 0.001, 0.01 and 0.1. Age, sex and Houseman-estimated white blood cell proportions [61] were incorporated as fixed effect covariates. The same settings as in the genetic analyses were applied. Methylation probes which had a posterior inclusion probability of $\geq 95 \%$ were deemed to be significant.

Details for the OLS and mixed linear model approaches are outlined in Additional file 3. For these methods, probes which surpassed a Bonferroni-corrected significance threshold of $5.14 \times 10^{-10}$ (= genome-wide significance $3.6 \times 10^{-8} / 70$ phenotypes) were deemed to be significant. The genome-wide significance level of $3.6 \times 10^{-8}$ was selected as per the recommendations of Safarri et al. [62].

\section{Functional annotation of genetic and epigenetic loci}

Genetic markers that were independently associated with protein levels were functionally annotated using ANNOVAR [63] and Ensembl genes (build 85) in FUMA (FUnctional Mapping and Annotation) [64]. Epigenetic probes associated with protein levels were annotated using the IlluminaHumanMethylation450kanno.ilmn12.hg19 package [65].

\section{Identification of overlap between cis pQTLs and cis eQTLs} To determine whether $\mathrm{PQTL}$ variants may affect protein levels through modulation of gene expression, we crossreferenced cis pQTLs with publicly available (and FDRcorrected significant) cis expression QTL (eQTL) data from the eQTLGen consortium. Expression QTL data were derived from blood tissue, $85 \%$ of samples were derived from whole blood and $15 \%$ of samples were derived from peripheral blood mononuclear cell data [66]. For each protein, expression QTLs were also subset to the gene (messenger RNA) encoding the protein of interest.

\section{Colocalisation}

To test whether a sole causal variant might underlie both an eQTL and pQTL association, we performed Bayesian tests of colocalisation using the coloc package in $\mathrm{R}$ [67]. For each protein of interest, a 200-kb region (upstream and downstream-recommended default setting) surrounding the appropriate pQTL was extracted from our GWAS summary statistics [68]. For each respective protein, the same region was also extracted from eQTLGen summary statistics. Default priors were applied. Summary statistics for all SNPs within these regions were used to determine the posterior probability for five distinct hypotheses: a single causal variant for both traits, no causal variant for either trait, a causal variant for one of the traits (encompassing two hypotheses), or distinct causal variants for the two traits. Posterior probabilities $(\mathrm{PP}) \geq 0.95$ provided strong evidence in favour of a given hypothesis.

\section{Pathway enrichment and tissue specificity analyses}

Using methylation data, pathway enrichment was assessed among KEGG pathways and Gene Ontology (GO) terms through hypergeometric tests using the phyper function in R. All gene symbols from the $450 \mathrm{~K}$ array annotation (null set of sites) were converted to Entrez IDs using biomaRt $[69,70]$. GO terms and their corresponding gene sets were retrieved from the Molecular Signatures Database (MSigDB)-C5 [71]. KEGG pathways were downloaded from the KEGG REST server [72]. Tissue specificity analyses were performed using the GENE2FUNC function in FUMA. Differentially expressed gene sets with Bonferroni-corrected $P$ values $<0.05$ and an absolute log-fold change of $\geq 0.58$ (default settings) were considered to be enriched in a given tissue type (GTEx v7).

\section{Mendelian randomisation}

Two-sample Mendelian randomisation was used to test for putatively causal relationships between (i) the 4 proteins whose pQTLs were previously shown to be associated with human traits, as identified through GWAS Catalog, and the respective traits [73, 74] (http://www. nealelab.is/uk-biobank/); (ii) the 13 proteins which harboured significant pQTLs and Alzheimer's disease risk [75]; (iii) gene expression and inflammatory protein levels; and (iv) DNA methylation and inflammatory protein levels. Pruned variants (LD $\left.r^{2}<0.1\right)$ were used as instrumental variables (IV) in MR analyses. In tests where only one independent SNP remained after LD pruning, causal effect estimates were assessed using the Wald ratio test, i.e. a ratio of effect per risk allele on trait to effect per risk allele on protein levels. In tests where multiple independent variants were identified, and if no evidence of directional pleiotropy was present (non-significant MR-Egger intercept), multi-SNP MR was carried out using inverse variance-weighted estimates. Analyses were conducted using MRbase [76]. Further details are provided in Additional file 3.

\section{Results}

Genome-wide studies of inflammatory protein levels

In a Bayesian penalised regression model (BayesR+), 16 pQTLs were identified for 14 proteins (Additional file 2: Table S2). Thirteen of these 16 pQTLs ( $n=13$ proteins) directly, or through variants in high linkage disequilibrium (LD) $r^{2}>0.75$, replicated conditionally significant pQTLs from the OLS regression model (Additional file 2: Tables S3-S5; Additional file 3). The correlation 


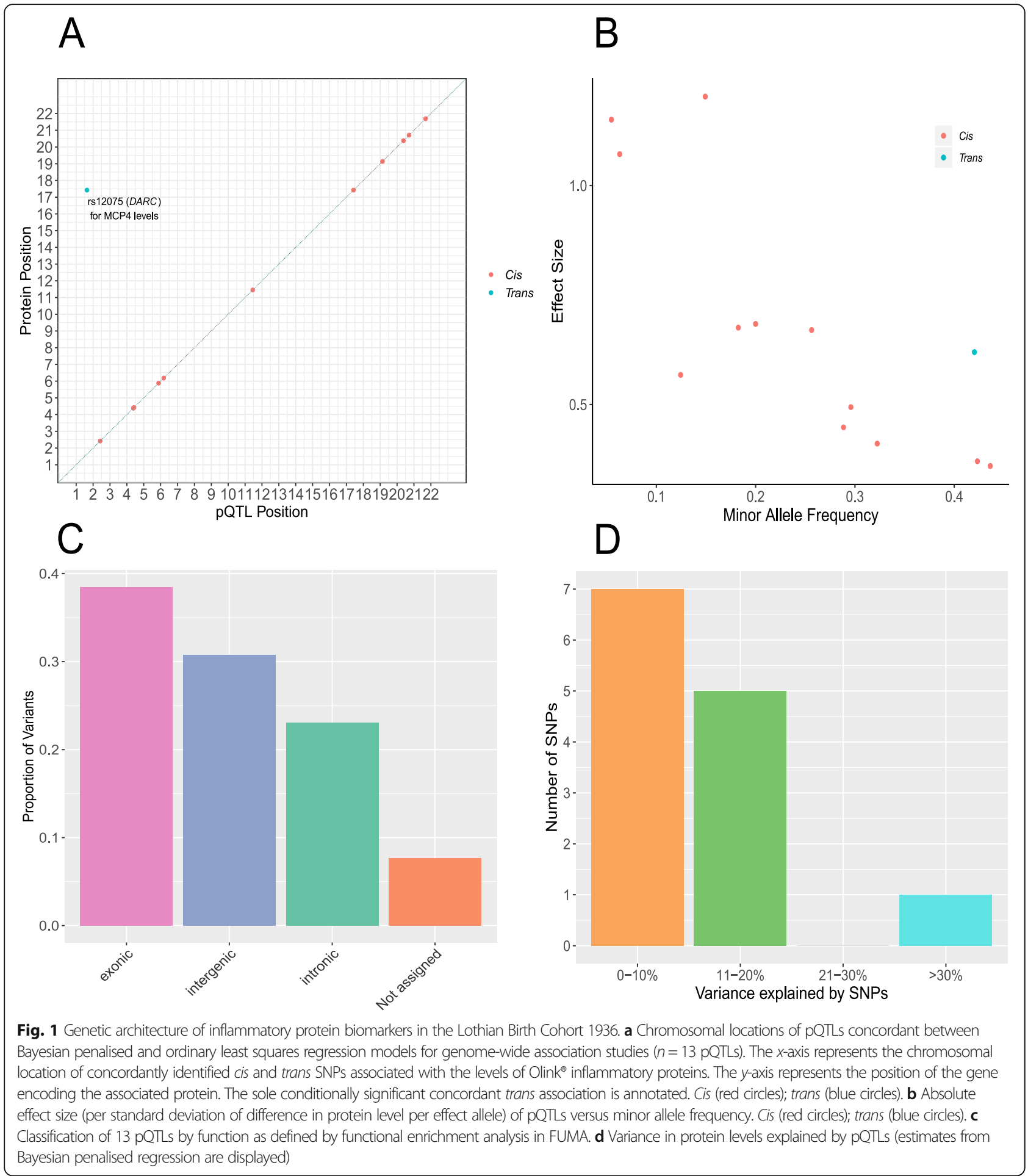

structure among these 13 proteins is shown in Additional file 4: Fig. S1.

Twelve (92.3\%) of the concordant SNPs were cis pQTLs (SNP within $10 \mathrm{Mb}$ of the transcription start site (TSS) of a given gene $[69,70])$ and 1 pQTL $(7.7 \%)$ was a trans-associated variant (Fig. 1a; Additional file 2: Table
S6). There was an inverse relationship between the minor allele frequency of variants and their effect size (Fig. 1b). The functional category to which the greatest proportion of variants was assigned was exonic variants (38.5\%), as identified by FUMA (FUnctional Mapping and Annotation analysis) (Fig. 1c). Four of the five SNPs 

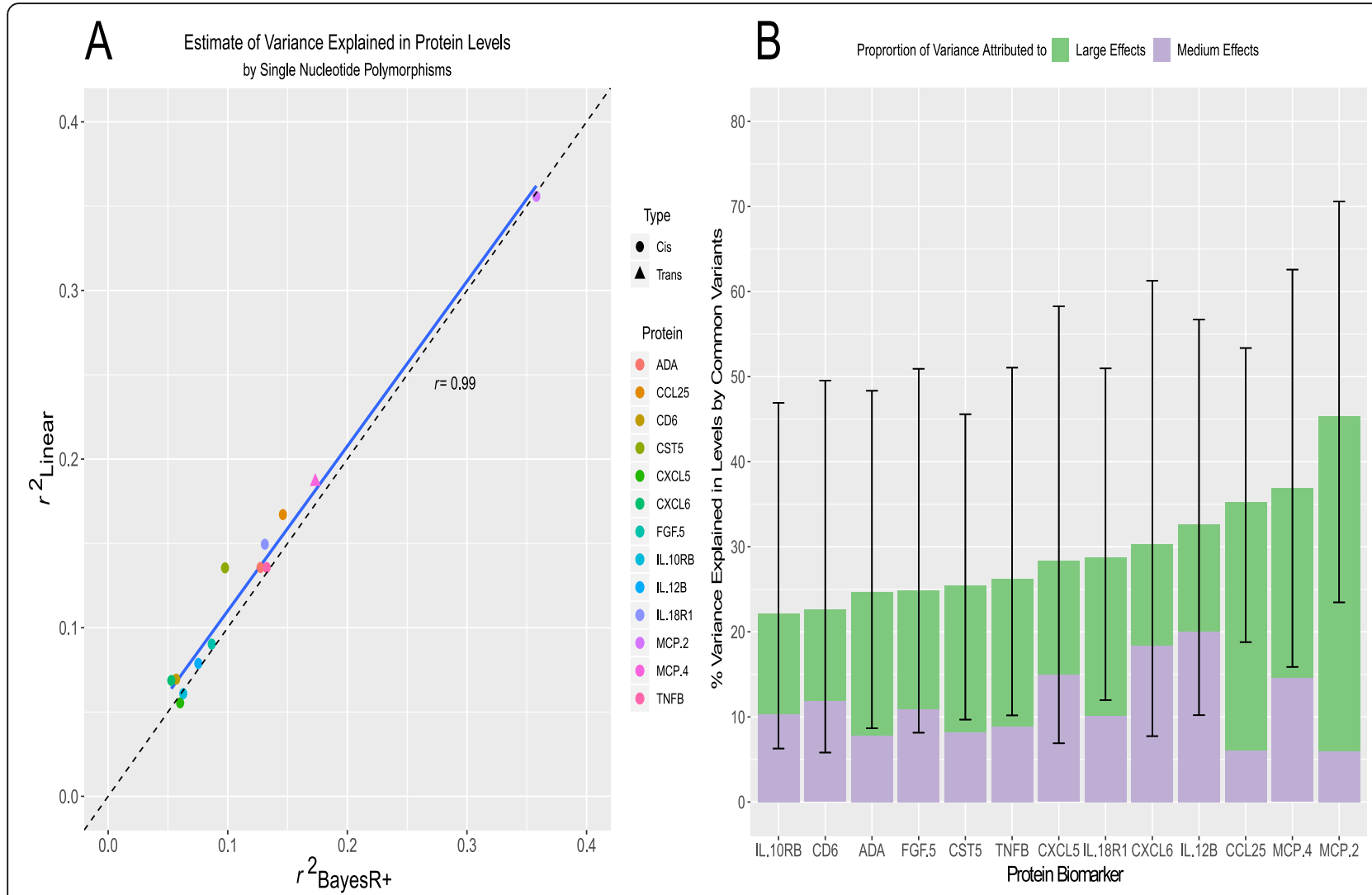

Fig. 2 Variance in circulating inflammatory protein levels explained by common genetic variation. a In this panel, the variance explained $\left(r^{2}\right)$ by consensus SNPS ( $n=13$ SNP, 1 per protein) in the ordinary least squares regression model was compared against the variance explained by the same SNP set identified in the Bayesian penalised regression approach. $\mathbf{b}$ The proportion of variance explained in Olink ${ }^{\otimes}$ inflammatory protein levels by common genetic variants genotyped in the LBC1936 participants is shown. Only those proteins which had significant PQTL associations in both the ordinary least squares and Bayesian methods are presented $(n=13)$. Additionally, the proportion of variance explained attributable to medium effects (prior: variance of 1\% explained) and large effects (prior: variance of 10\% explained) are demonstrated in purple and green,

respectively. Error bars represent 95\% credible intervals

annotated to exonic regions produce missense mutations. From the Bayesian model, pQTLs explained between 5.28\% (rs10005565; CXCL6) and 35.80\% (rs3138036; MCP2) of inter-individual variation in protein levels (Fig. 1d). The estimates for variance accounted for in protein levels by single SNPs were correlated 99\% between the BayesR+ and OLS regression models (Fig. 2a; Additional file 2: Table S6). The BayesR+ common (minor allele frequency $>1 \%$ ) SNPbased heritability estimates ranged from 11.4\% (CXCL9; $95 \%$ credible interval $[0 \%, 43.5 \%])$ to $45.3 \%$ (MCP2; $95 \%$ credible interval: $[23.5 \%, 70.6 \%])$, with a mean estimate of $20.2 \%$ across the 70 proteins (Additional file 2: Table S7). Figure $2 b$ shows heritability estimates for the 13 proteins exhibiting concordantly identified pQTLs across OLS regression and Bayesian approaches. Figure 3 demonstrates the effect of genetic variation at the most significant cis pQTL (rs3138036; MCP2) and the sole trans pQTL (rs12075; MCP4) on protein levels.
There was a strong correlation between our SNPbased heritability estimates and those from a previous study of 961 individuals [44]: 29 overlapping proteins, $r$ $0.71,95 \%$ CI $[0.43,0.84]$ (Additional file 2: Table S8 and Additional file 4: Fig. S2).

\section{Molecular mechanisms underlying pQTLs: colocalisation analysis}

Of the 12 cis pQTLs which were identified across OLS regression and BayesR+, 8 SNPs (66.67\%) previously have been identified as cis-acting expression QTLs (eQTLs) in blood (Additional file 2: Table S9). Using coloc [67], we tested the hypothesis that one causal variant might underlie both a pQTL and eQTL for each protein. For $4 / 8$ proteins, there was strong evidence (posterior probability $(\mathrm{PP})>0.95$ ) for colocalisation of $c$ is pQTLs and cis eQTLs (Additional file 2: Table S10). These proteins were CCL25, CD6, CXCL5 and CXCL6. 


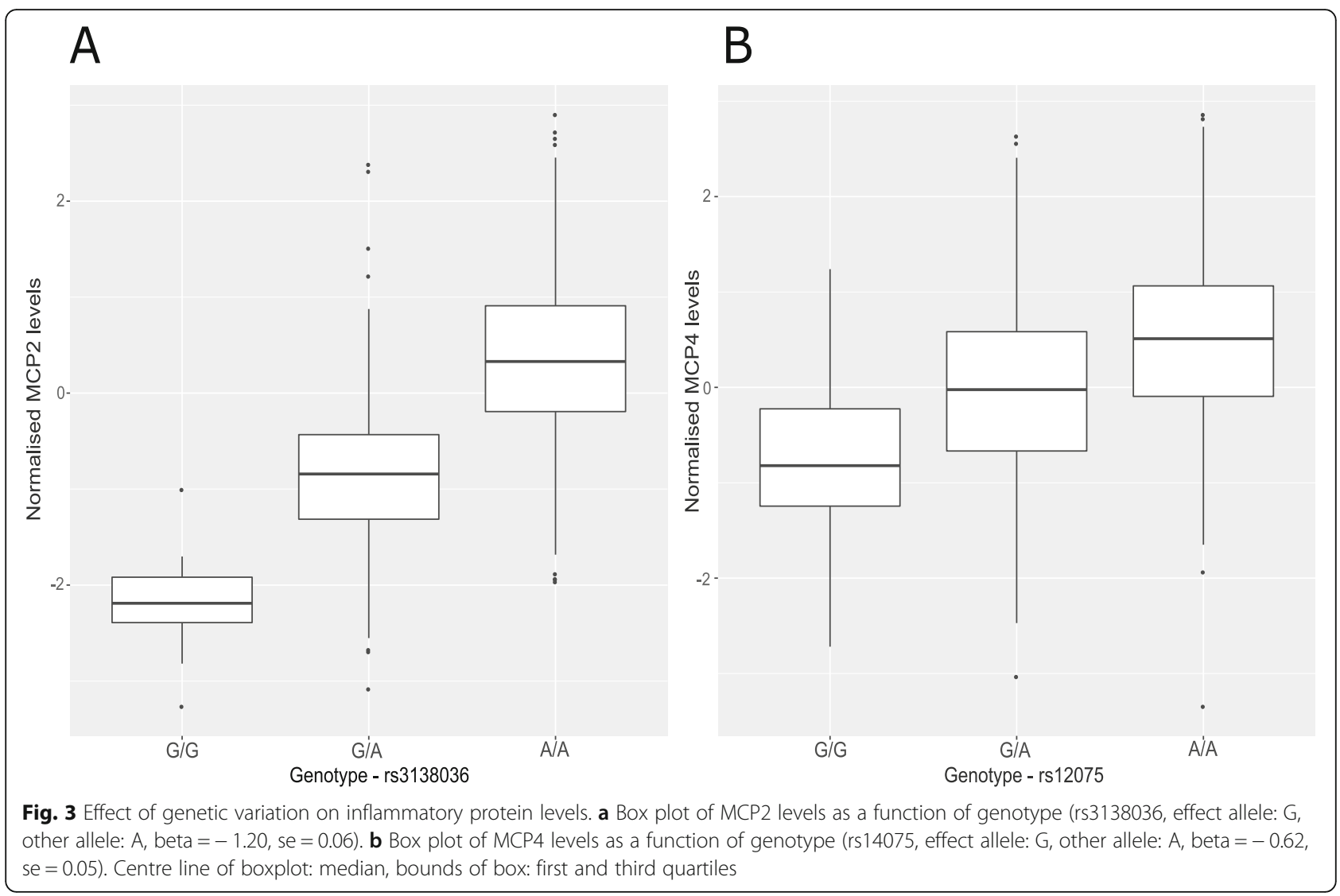

Mendelian randomisation analyses (MR; see the 'Methods' section) indicated that altered gene expression was causally associated with changes in protein levels for each of the four aforementioned proteins (CCL25, CD6, CXCL5 and CXCL6; range of beta [0.68, 12.25], se [0.09, $\left.1.12], P\left[9.54 \times 10^{-7}, 1.05 \times 10^{-37}\right]\right)$. However, a second colocalisation approach termed Sherlock [77] suggested that, from the 13 proteins with concordantly identified pQTLs, only expression of ADA, CXCL5 and IL18R1 were associated with levels of their respective protein products (Additional file 2: Table S11; Additional file 3).

\section{Epigenome-wide studies of inflammatory protein levels}

In the Bayesian model, 8 CpG-protein associations $(n=8$ proteins) had a posterior inclusion probability of more than 95\% (Additional file 2: Table S12). Five of these associations overlapped with those identified by the OLS regression model $\left(P<5.14 \times 10^{-10}\right.$; Additional file 2: Table S13); three of which were also identified in the mixed model approach $\left(P<5.14 \times 10^{-10}\right.$; Additional file 2: Table S14). These were the smoking-associated probe cg05575921 for CCL11 levels (trans association at AHRR; mixed model-beta -1.97 , se $\left.0.32, \quad P 4.86 \times 10^{-10}\right)$, cg07839457 for CXCL9 levels (trans association at
NLRC5; beta -2.91 , se $\left.0.39, \quad P \quad 8.03 \times 10^{-14}\right)$ and cg03938978 for IL18R1 levels (cis association at IL18RAP; beta -1.37 , se $0.16, P 5.86 \times 10^{-17}$ ) (Additional file 2 : Table S14). Adjustment for smoking attenuated the association between CCL11 levels and the cg05575921 probe (linear model-before adjustment: beta $-1.74, P 2.68 \times$ $10^{-10}$, after adjustment: beta $-1.20, P 0.03$; \% attenuation 31.03\%). GWAS and EWAS of CCL11 levels were repeated adjusting for smoking status, the results of the association studies are detailed in Additional file 3. Figure 4 depicts an epigenetic map of CpG-protein associations within this study and demonstrates the degree of overlap between methodologies. The correlation among the three proteins with concordantly identified CpG associations is shown in Additional file 4: Fig. S3. Look-up analyses of the top GWAS and EWAS findings with those reported in the literature are detailed in Additional file 3. For the GWAS, 11/13 pQTLs (84.62\%) from the present study were previously reported in the literature. The two loci which represent novel pQTLs are rs11700291 (ADA) and rs1458038 (FGF-5). Beta coefficients displayed a correlation coefficient of 0.88 between those in the present study and those reported in previous studies. For the EWAS, only one of the three concordantly identified 


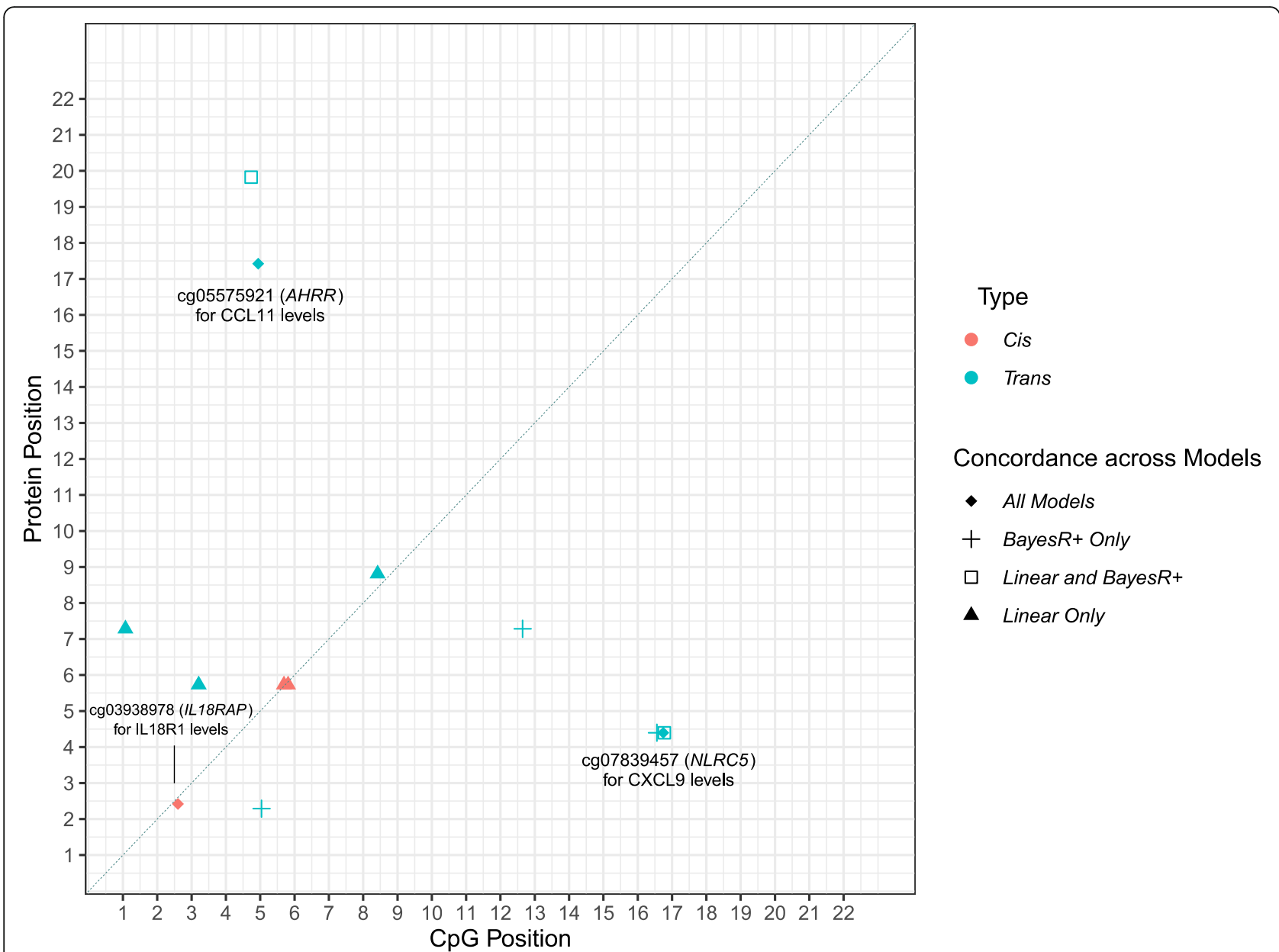

Fig. 4 Genomic locations of $\mathrm{CpG}$ sites associated with differential inflammatory protein levels. The $x$-axis represents the chromosomal location of $\mathrm{CpG}$ sites associated with the levels of Olink $k^{\circledR}$ inflammation biomarkers. The $y$-axis represents the position of the gene encoding the associated protein. The level of concordance across three models used to perform epigenome-wide association studies is represented by different shape patterns. Those CpG sites $(n=3)$ which were identified by linear (ordinary least squares), mixed model and Bayesian penalised regression models, and passed a Bonferroni-corrected significance threshold are represented by diamonds and annotated. Three proteins (CXCL9, CXCL10 and CXCL11) were associated with differential methylation levels at the cg07839457 site in the NLRC5 transcription factor locus. Additionally, two proteins (CCL11 and TGF-alpha) were associated with the smoking-associated cg05575921 site in the AHRR locus. Cis (red); trans (blue)

CpG-protein associations was previously reported in the literature by Ahsan et al. [44]. This association was between the cg07839457 probe (NLRC5) and CXCL9 levels (beta $_{\mathrm{LBC}}-2.91$ vs. beta Ahsan -3.26 ).

We conducted tissue specificity and pathway enrichment analyses based on genes identified by EWAS for each of the 3 proteins with significant CpG associations. Tissue-specific patterns of expression were observed for $2 / 3$ proteins (Additional file 4: Fig. S4-S6). For CCL11, differential expression was observed in breast, adipose and kidney tissue. For IL18R1, differential expression of associated genes was observed in pancreatic tissue. Furthermore, down-regulation of genes associated with IL18R1 was observed in the hippocampus and substantia nigra. There was no significant enrichment of pathways incorporating genes annotated to CXCL9, CCL11 or IL18R1 following multiple testing correction.
One protein, IL18R1, harboured both a significant cis pQTL and cis CpG site in our study (Additional file 4: Fig. S7). This SNP (rs917997) previously has been identified as a methylation QTL (mQTL) for the single cis CPG site associated with IL18R1 levels identified by our epigenome-wide studies (cg03938978) [78]. Using bidirectional MR analysis (Wald ratio test; see methods), we show evidence that DNA methylation at this locus may be causally associated with circulating IL18R1 levels (beta -0.81 , se $0.17, P 2.14 \times 10^{-33}$ ). Conversely, IL18R1 levels may also be causally associated with altered DNA methylation (beta -1.22 , se $0.16, P 3.4 \times 10^{-14}$ ).

The methylation data explained an average of $18.2 \%$ of variance in protein levels using BayesR+; estimates ranged from $6.3 \%$ (IL15RA, 95\% credible interval [0.0\%, $27.3 \%]$ ) to $46.1 \%$ (CXCL10, 95\% credible interval [24.1\%, 

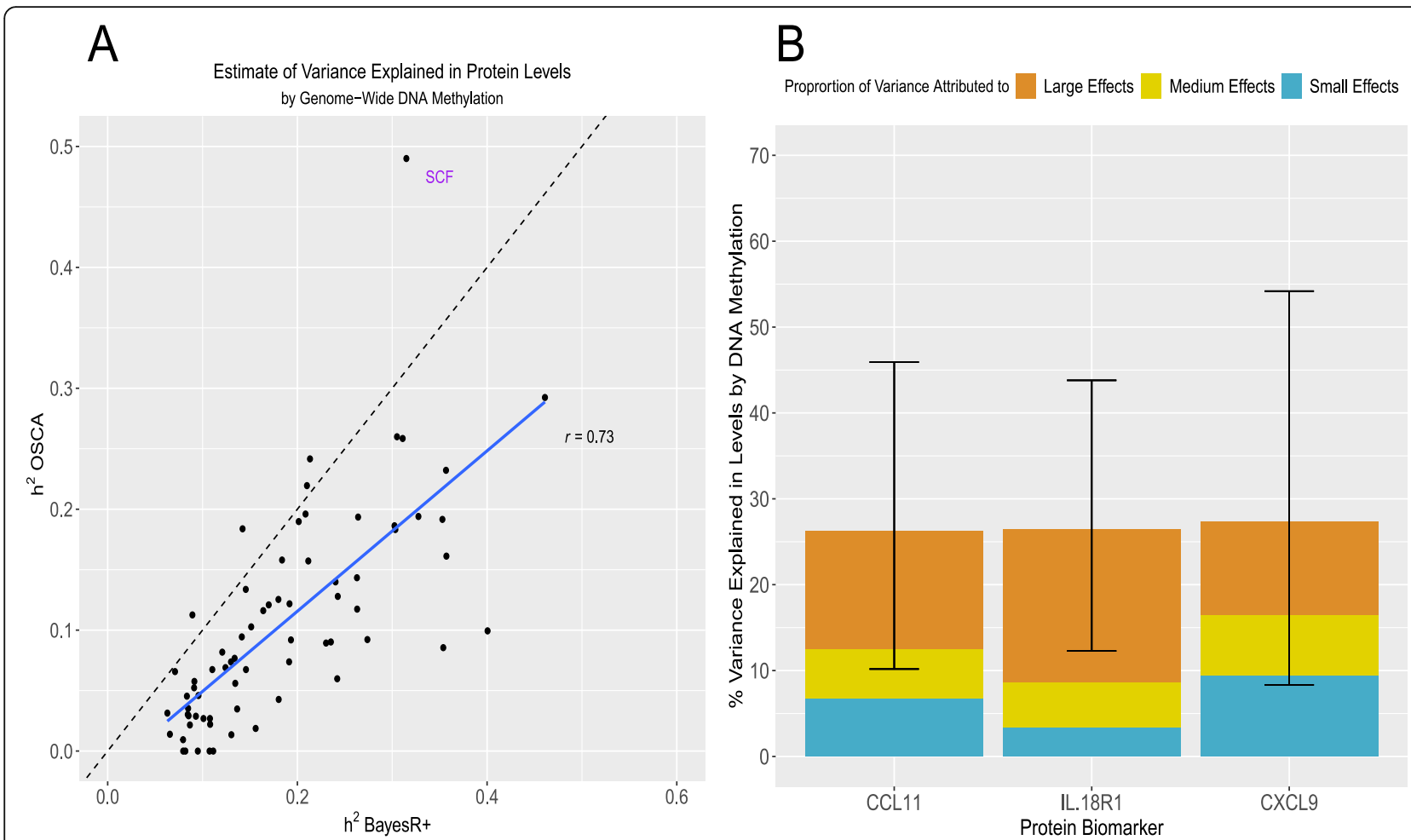

Fig. 5 Variance in circulating inflammatory protein levels explained by DNA methylation. a In this panel, the variance explained in circulating protein levels by complete methylation data from sites present on the Infinium $450 \mathrm{~K}$ methylation array was examined. A comparison between variance explained $\left(\mathrm{h}^{2}\right)$ by a mixed model approach (OSCA) and a Bayesian penalised regression approach (BayesR+) is shown. $\mathbf{b}$ The proportion of variance explained in Olink ${ }^{\circledR}$ inflammatory protein levels by DNA methylation, as estimated by BayesR+ is shown. Only those proteins ( $n=3$ ) which had significant CpG associations in ordinary least squares, mixed model and Bayesian methods are presented. Additionally, the proportion of variance explained attributable to small effects (prior: variance of $0.1 \%$ explained), medium effects (prior: variance of $1.0 \%$ explained) and large effects (prior: variance of 10\% explained) are demonstrated in blue, gold and dark orange, respectively. Error bars represent $95 \%$ credible intervals

67.1\%]) (Additional file 2: Table S15). There was strong concordance with estimates from the mixed model sensitivity analysis (Additional file 2: Table S16 and Fig. 5a). Figure $5 \mathrm{~b}$ shows the variance explained by methylation data for the 3 proteins exhibiting concordantly identified CpGs across OLS regression, mixed-model and Bayesian approaches.

\section{Variation in inflammatory protein levels explained by genetics and DNA methylation}

When accounting for genetic data, the estimates for variance explained by methylation data were largely unchanged for most proteins (Additional file 2: Table S17; $n=9$ proteins with change $>5 \%, 1$ with change $<-5 \%$ (VEGFA)). The mean absolute change was $2.6 \%$ (minimum $0.01 \%$ for TNFRSF9 and maximum $15.0 \%$ for IL18R1). Similarly, estimates from genetic data were largely unchanged in the combined analysis $(n=2$ proteins with change $>5 \%$ ). The mean absolute change was $1.8 \%$ (minimum $0.02 \%$ for CD244 and maximum $6.7 \%$ for CCL28). For 22 proteins, the variance explained by methylation data was greater than that explained by genetic data (Additional file 5).
For each protein, we performed $t$-tests to determine whether the variance explained by methylation or genetic data alone was significantly different from the estimate for variance explained in the combined analysis. For methylation data, 40 proteins showed a significant difference between the estimates for variance in protein levels explained by methylation data alone and methylation data conditional on SNPs $(P<0.05)$. For genetic data, 50 proteins showed a significant difference $(P<$ 0.05) (Additional file 2: Table S17).

The combined estimate for variance explained by genetic and methylation data ranged from $23.4 \%$ for CXCL1 to $66.4 \%$ for VEGFA. The mean and median estimates were 37.7 and $36.0 \%$, respectively. Details of which SNPs and CpGs were identified as being associated with protein levels in the combined BayesR+ analyses, accounting for all genetic and epigenetic factors together, is outlined in Additional file 2: Table S18 and Additional file 3.

\section{Evaluating causal associations between inflammatory biomarkers and human traits}

The 13 independent pQTL associations were queried against GWAS Catalog to identify existing associations 
between these pQTLs and phenotypes [73]. We investigated whether these associations represented causal relationships. Using two-sample MR, we showed that CD6 levels were causally associated with inflammatory bowel disease (IBD) (beta 0.20 , se $0.04, P 2.59 \times 10^{-6}$ ). Furthermore, FGF-5 levels were causally associated with systolic and diastolic blood pressure (beta 0.07 and 0.07 , se 0.01 and $0.01, P 1.04 \times 10^{-34}$ and $4.29 \times 10^{-42}$, respectively). IL12B levels were associated with Crohn's disease (beta 0.42 , se $\left.0.05, P 2.76 \times 10^{-15}\right)$. Circulating IL18R1 levels showed a causal relationship with IBD (beta 0.17 , se 0.03, $\left.P 1.63 \times 10^{-9}\right)$.

Peripheral inflammatory processes and proteins have been linked to risk of late-onset Alzheimer's disease (AD) $[79,80]$. We tested whether the 13 proteins with significant genetic correlates in our study were causally associated with AD risk (Additional file 3). One protein, IL18R1, showed a nominally significant, unidirectional relationship with $\mathrm{AD}$ risk (beta 0.02, se 0.01, $P$ 0.04) (Additional file 2: Table S19).

\section{Discussion}

Using a Bayesian framework and sensitivity analyses with OLS regression and mixed linear models, we robustly identified 13 independent genetic and 3 epigenetic correlates of circulating inflammatory protein levels. Two of these pQTLs and two CpG sites have not been previously reported as genome-wide significant in the literature. This is the first study to have integrated genetic and epigenetic data together using multiple methods to identify molecular correlates of, and estimate the contribution of these molecular factors towards interindividual variability in, the circulating proteome. Our results also provide an important and novel demonstration of the overlap between disparate methodologies for performing genome-wide and epigenome-wide association studies on proteomic data. Using integrative causal frameworks, we identified mechanisms through which genetic variation may perturb plasma protein levels. Additionally, we demonstrated causal relationships between prioritised circulating inflammatory proteins and blood pressure as well as inflammatory bowel diseases.

For genome-wide association studies, there is a necessity to perform secondary analyses in order to identify independent loci from association studies. This is often carried out through employing conditional and joint analyses (GCTA-COJO) or LD clumping-based methods, such as those implemented in FUMA [54, 64]. BayesR+ negates the need for such secondary analyses; it allows for the modelling of single marker or probe effects whilst controlling for all other markers or probes. Indeed, BayesR+ can outperform OLS regression or mixed model methods in providing single probe or marker coefficient estimates whilst controlling for all other input
SNP and/or CpG sites, as well as known and unknown confounding variables. However, identifying true molecular correlates of protein data over false positive associations is challenging. By relying on careful corrections for multiple testing and triangulation of evidence across disparate methods, our stringent approach was wellequipped to identify likely true biological signal as opposed to false positives.

The issue of identifying true biological signals over false positive associations is particularly pertinent in relation to trans associations which show poor replication and often have smaller effect sizes than cis associations [81]. We identified one trans pQTL (rs12075) associated with levels of the chemokine MCP4 (encoded for by CCL13 gene on chromosome 17). This SNP represents a nonsynonymous polymorphism (Asp42Gly) annotated to the Duffy antigen/chemokine receptor (DARC) gene on chromosome 1. Previously, this SNP has been associated with lower MCP1 levels and evidence shows that the base-change results in altered chemokine-receptor binding $[10,20,82]$. Additionally, this polymorphism has been shown to explain approximately $20 \%$ of variation in MCP1 levels, similar to our estimate of $18.66 \%$ in MCP4 levels [82]. The Duffy antigen receptor is expressed on erythrocytes and acts as a reservoir for circulating chemokines resulting in reduced distribution of chemokines to extravascular tissue and dampened pro-inflammatory effects [83]. Our findings suggest that this polymorphism may also lead to reduced MCP4 levels, possibly through augmented chemokine-receptor interaction.

In the EWAS analyses, the probe cg05575921, located in the AHRR locus, was associated with CCL11 levels. This probe is strongly associated with smoking status [84-91] and the association was attenuated after adjustment for smoking. Furthermore, higher levels of CCL11 have been associated with tobacco smoking and cannabis use [92-94]. We also found altered methylation at the NLRC5 locus (NOD-like receptor family CARD domain containing 5) is associated with circulating CXCL9 levels. NLRC5 acts as a potent regulator of the inflammasome [44, 95]. Zaghlool et al. showed that altered methylation at the NLRC5 locus associates with several inflammatory markers, including CXCL10 and CXCL11, with pathway analyses linking it to disease states in which NLRC5 dysfunction is implicated such as cancer and cardiovascular disease [43].

Using our database of genotype-protein associations, we tested for causal relationships between inflammatory protein biomarkers and human phenotypes. However, in each case, only one variant was available to test for such associations which does not allow for the testing of pleiotropic effects. CD6 was associated with clinically diagnosed IBD. Expression of the CD6 receptor and its ligand, ALCAM, are overexpressed in the intestinal mucosa of IBD patients 
where it may promote $\mathrm{CD} 4^{+} \mathrm{T}$ cell proliferation and differentiation into pro-inflammatory Th1/Th17 cells [96]. FGF-5 levels were associated with automated readings of systolic and diastolic pressure; previously, FGF-5 levels have been significantly correlated with blood pressure [97]. Variation in the IL12B gene has been linked strongly to the pathogenesis of Crohn's disease and an antibody targeted towards the p40 subunit of IL12 demonstrated efficacy in the treatment of moderate-to-severe Crohn's disease [98]. In our study, we showed that circulating IL12B levels may be causally linked to this disease. Lastly, IL18R1 levels may also be causally associated with IBD. A number of studies have demonstrated that increased IL18 signalling confers detrimental effects in the context of gastrointestinal inflammatory processes [99].

Our study has a number of caveats. First, proteins with high sequence homology and structural similarities to a targeted protein of interest may be inappropriately captured by assay probes resulting in quantification errors. Olink ${ }^{\oplus}$ 's Proximity Extension Assay technology uses a matched pair of antibodies, coupled to unique, partially complementary oligonucleotides resulting in exceptional readout specificity and greatly reducing this problem compared to other immunoassays. Second, there was a strong correlation structure among the inflammatory protein panel. However, given that inflammatory proteins are often co-expressed and synergistic, overlapping loci may reveal biologically important foci or nodes of co-regulation [100]. Third, functional enrichment analyses indicated that four robustly identified pQTL signals reflect missense mutations in their protein products, three of which were cis associations with proteins present on the Olink $^{\circ}$ inflammation panel. This may lead to altered structural properties of the protein target, thereby affecting antibody-antigen recognition and the ability of assays to accurately quantify protein levels. It is possible that the variants identified may not reflect variants causally associated with blood protein levels, and instead capture a causal variant in the locus. Nevertheless, the identification of such potential protein-altering variants is an important technical consideration in studies aiming to determine the molecular architecture of the human proteome. Furthermore, these variants reflect important candidates for functional characterisation in in vitro studies which aim to dissect their influence on protein abundance in cellular systems. Fourth, our Scottish cohort contains individuals from a homogenous genetic background limiting the generalisability of our findings to individuals of other ethnic backgrounds. Fifth, ageing is closely linked to chronic low-grade inflammation. Therefore, the distributions of, and correlation structure among, inflammatory protein biomarkers may differ in our cohort of healthy older ageing when compared to other age ranges and the general older adult population. Sixth, the sample size within our study resulted in large confidence and credible intervals in the reported estimates for heritabilities in inflammatory protein levels.

\section{Conclusions}

Our integrative and multi-method approach has identified high-confidence genetic and epigenetic loci associated with inflammatory protein biomarker levels. Furthermore, we have provided novel estimates for the contribution of common genetic and epigenetic variation towards differences in circulating inflammatory biomarker levels, considered alone and together. Together, our data may have important implications for informing the molecular regulation of the human proteome. Our data provides a platform upon which other researchers may investigate relationships between inflammatory biomarkers and disease, and a resource to further inform biological insights into immunological and inflammatory processes.

\section{Supplementary information}

Supplementary information accompanies this paper at https://doi.org/10. 1186/s13073-020-00754-1.

\section{Additional file 1. Distribution of raw values for inflammatory protein} levels across individuals in Lothian Birth Cohort 1936.

Additional file 2: Supplementary Tables. The association of preadjusted protein levels with biological and technical covariates. Protein levels were adjusted for age, sex, array plate and four genetic principal components (population structure) prior to analyses. Significant associations are emboldened. (Table S1). PQTLs associated with inflammatory biomarker levels from Bayesian penalised regression model (Posterior Inclusion Probability > 95\%). (Table S2). All pQTLs associated with inflammatory biomarker levels from ordinary least squares regression model $\left(\mathrm{P}<7.14 \times 10^{-10}\right)$. (Table S3). Summary of lambda values relating to or dinary least squares GWAS and EWAS performed on inflammatory protein levels $(n=70)$ in Lothian Birth Cohort 1936 study. (Table S4). Conditionally significant PQTLs associated with inflammatory biomarker levels from ordinary least squares regression model $\left(P<7.14 \times 10^{-10}\right)$. (Table S5) Comparison of variance explained by ordinary least squares and Bayesian penalised regression models for concordantly identified SNPs. (Table S6) Estimate of heritability for blood protein levels as well as proportion of variance explained attributable to different prior mixtures. (Table S7). Comparison of heritability estimates from Ahsan et al. (maximum likelihood) and Hillary et al. (Bayesian penalised regression). (Table S8). List of concordant SNPs identified by linear model and Bayesian penalised regression and whether they have been previously identified as eQTLs. (Table S9). Bayesian tests of colocalisation for cis PQTLs and cis eQTLs. (Table S10). Sherlock algorithm: Genes whose expression are putatively associated with circulating inflammatory proteins that harbour PQTLS. (Table S11). CpGs associated with inflammatory protein biomarkers as identified by Bayesian model (Bayesian model; Posterior Inclusion Probability $>$ 95\%). (Table S12). CpGs associated with inflammatory protein biomarkers as identified by linear model (limma) at $P<5.14 \times 10^{-10}$.

(Table S13). CpGs associated with inflammatory protein biomarkers as identified by mixed linear model (OSCA) at $P<5.14 \times 10^{-10}$. (Table S14) Estimate of variance explained for blood protein levels by DNA methylation as well as proportion of explained attributable to different prior mixtures - BayesR+. (Table S15). Comparison of variance in protein levels explained by genome-wide DNA methylation data by mixed linear model (OSCA) and Bayesian penalised regression model (BayesR+). (Table S16). Variance in circulating inflammatory protein biomarker levels explained 
by common genetic and methylation data (joint and conditional estimates from BayesR+). Ordered by combined variance explained by genetic and epigenetic data - smallest to largest. Significant results from ttests comparing distributions for variance explained by methylation or genetics alone versus combined estimate are emboldened. (Table S17). Genetic and epigenetic factors identified by BayesR+ when conditioning on all SNPs and CpGs together. (Table S18). Mendelian Randomisation analyses to assess whether proteins with concordantly identified genetic signals are causally associated with Alzheimer's disease risk. (Table S19).

Additional file 3. Details of Supplementary Methods. Contains information for the following data: Conditional and joint analysis from ordinary least squares GWAS on protein levels; Sherlock: identifying genes whose expression associates with inflammatory biomarkers; GWAS and EWAS of CCL11 levels - incorporating smoking status as a covariate; Replication of previous PQTLs and protein associated-CpG sites; BayesR+ combined analysis - GWAS and EWAS modelled together; Evaluating causal associations between blood inflammatory proteins and Alzheimer's risk.

Additional file 4: Supplementary Figures. Correlation between the 13 proteins with significant $\mathrm{PQTLS}$ as identified by ordinary least squares and Bayesian penalised regression. (Figure S1). Correlation between heritability estimates for circulating inflammatory protein biomarkers from present study and that of Ahsan et al. The protein with the greatest discordance between studies (MMP-1) is annotated. (Figure S2). Correlation between the 3 proteins with significant $\mathrm{CpG}$ associations as identified across ordinary least squares model, mixed model and Bayesian penalised regression approaches. (Figure S3). Tissue-specific expression of genes annotated to CpGs associated with CCL11 levels at $P<1 \times 10^{-5}$. Differential expression was observed in kidney, adipose and breast tissue. (Figure S4). Tissue-specific expression of genes annotated to CpGs associated with IL18R1 levels at $P<1 \times 10^{-5}$. Differential expression was observed in pancreatic, hippocampal and substantia nigra tissue. (Figure S5). Tissue-specific expression of genes annotated to CpGs associated with CXCL9 levels at $P<1 \times 10-5$. No tissue-specific expression was observed. (Figure S6). Miami plot for IL18R1 which exhibited both genome-wide significant SNP and genome-wide significant CpG associations. The top half of the plot (skyline) shows the results from the GWAS on protein levels, whereas the bottom half (waterfront) shows the results from the EWAS. IL18R1 (chromosome 2: 102,311,529-102,398,775). (Figure S7).

Additional file 5. Variance in circulating protein levels explained by common genetic and methylation data together.

\section{Acknowledgements}

The authors thank LBC1936 study participants and research team members who have contributed, and continue to contribute, to ongoing LBC1936 studies.

\section{Authors' contributions}

R.F.H, M.R.R. and R.E.M were responsible for the conception and design of the study. R.F.H carried out the data analyses. R.F.H, M.R.R and R.E.M drafted the article. D.T.B, A.K, D.L.MC.C., Q.Z, D.C.L and S.E.H contributed to the data preparation. S.E.H, N.R.W, A.F.M, P.M.V and I.J.D were responsible for the data collection. All authors read and approved the final manuscript.

\section{Funding}

The LBC1936 is supported by Age UK (Disconnected Mind program, which supports S.E.H), the Medical Research Council (MR/M01311/1), and the University of Edinburgh. Genotyping was supported by the Biotechnology and Biological Sciences Research Council (BB/F019394/1). Methylation typing was supported by Centre for Cognitive Ageing and Cognitive Epidemiology (Pilot Fund award), Age UK, The Wellcome Trust Institutional Strategic Support Fund, The University of Edinburgh, and The University of Queensland. Proteomic analyses were supported for by the LBC1936 Age UK grant. This work was conducted in the Centre for Cognitive Ageing and Cognitive Epidemiology, which was supported by the Medical Research Council and Biotechnology and Biological Sciences Research Council (MR/ K026992/1), and which supported I.J.D. We acknowledge NIH Grants R01AG054628 and R01AG05462802S1 for supporting this research and Grant
P2CHD042849 for supporting the Population Research Center at the University of Texas. R.F.H. and A.J.S. are supported by funding from the Wellcome Trust 4-year PhD in Translational Neuroscience-training the next generation of basic neuroscientists to embrace clinical research [R.F.H: 108890/Z/15/Z; A.J.S: 203771/Z/16/Z]. D.L.Mc.C. and R.E.M. are supported by Alzheimer's Research UK major project grant ARUK-PG2017B-10. This research was supported by Australian National Health and Medical Research Council (grants 1010374, 1046880 and 1113400) and by the Australian Research Council (DP160102400). P.M.V., N.R.W. and A.F.M. are supported by the NHMRC Fellowship Scheme (1078037, 1078901 and 1083656). P.M.V was also funded by the Australian Research Council (DP160102400 and FL180100072).

\section{Availability of data and materials}

Lothian Birth Cohort 1936 data are available on request from the Lothian Birth Cohort Study, Centre for Cognitive Ageing and Cognitive Epidemiology, University of Edinburgh. Lothian Birth Cohort 1936 data are not publicly available due to them containing information that could compromise participant consent and confidentiality.

Full and openly accessible summary statistics from the association studies on Olink inflammatory protein levels are available on the University of Edinburgh Datashare site (https://datashare.is.ed.ac.uk/). These data pertain to summary statistics for GWAS (performed by two methods) and EWAS (performed by three methods) on the levels of 70 inflammatory proteins measured in members of the Lothian Birth Cohort 1936. For OLS regression GWAS data, see https://datashare.is.ed.ac.uk/handle/10283/3624; https://doi org/10.7488/ds/2814 [101]. For BayesR+ GWAS data, see https://datashare.is ed.ac.uk/handle/10283/3673; https://doi.org/10.7488/ds/2854 [102]. For OLS regression EWAS data, see https://datashare.is.ed.ac.uk/handle/10283/3628, https://doi.org/10.7488/ds/2818 [103]. For OSCA EWAS data, see https:// datashare.is.ed.ac.uk/handle/10283/3627, https://doi.org/10.7488/ds/2817 [104]. For BayesR+ EWAS data, see https://datashare.is.ed.ac.uk/handle/102 83/3626; https://doi.org/10.7488/ds/2816 [105]. Summary statistics for the OLS GWAS data are also available at GWAS Catalog (https://www.ebi.ac.uk/ gwas/; Study Accessions: GCST90000437-GCST90000506) [106].

\section{Ethics approval and consent to participate}

Ethical permission for the LBC1936 was obtained from the Multi-Centre Research Ethics Committee for Scotland (MREC/01/0/56) and the Lothian Research Ethics Committee (LREC/2003/2/29). Written informed consent was obtained from all participants. This study was performed in accordance with the Helsinki declaration.

\section{Consent for publication}

Not applicable.

\section{Competing interests}

The authors declare that they have no competing interests.

\section{Author details}

${ }^{1}$ Centre for Genomic and Experimental Medicine, Institute of Genetics and Molecular Medicine, University of Edinburgh, Edinburgh EH4 2XU, UK. ${ }^{2}$ Department of Computational Biology, University of Lausanne, 1015 Lausanne, Switzerland. ${ }^{3}$ Department of Psychology, University of Edinburgh, Edinburgh EH8 9JZ, UK. ${ }^{4}$ Lothian Birth Cohorts, University of Edinburgh, Edinburgh EH8 9JZ, UK. Institute for Molecular Bioscience, University of Queensland, Brisbane, Queensland 4072, Australia. ${ }^{6}$ Edinburgh Dementia Prevention, Centre for Clinical Brain Sciences, University of Edinburgh, Edinburgh EH16 4UX, UK. ${ }^{7}$ Department of Psychology, The University of Texas at Austin, Austin, TX 78712, USA. ${ }^{8}$ Population Research Center, The University of Texas at Austin, Austin, TX 78712, USA. 'Institute of Science and Technology Austria, 3400 Klosterneuburg, Austria.

Received: 11 March 2020 Accepted: 10 June 2020

Published online: 08 July 2020

\section{References}

1. Chen L, Deng H, Cui H, Fang J, Zuo Z, Deng J, et al. Inflammatory responses and inflammation-associated diseases in organs. Oncotarget. 2017;9(6): 7204-18.

2. Murakami M, Hirano T. The molecular mechanisms of chronic inflammation development. Fronti immunol. 2012;3:323. 
3. Furman D, Campisi J, Verdin E, Carrera-Bastos P, Targ S, Franceschi C, et al. Chronic inflammation in the etiology of disease across the life span. Nat Med. 2019;25(12):1822-32.

4. Amor S, Puentes F, Baker D, van der Valk P. Inflammation in neurodegenerative diseases. Immunology. 2010;129(2):154-69.

5. Pahwa R, Goyal A, Bansal P, Jialal I. Chronic Inflammation. Treasure Island (Florida): StatPearls Publishing; 2020

6. Ligthart S, Vaez A, Vosa U, Stathopoulou MG, de Vries PS, Prins BP, et al. Genome analyses of $>200,000$ individuals identify 58 loci for chronic inflammation and highlight pathways that link inflammation and complex disorders. Am J Hum Genet. 2018;103(5):691-706.

7. Dehghan A, Dupuis J, Barbalic M, Bis JC, Eiriksdottir G, Lu C, et al. Metaanalysis of genome-wide association studies in $>80000$ subjects identifies multiple loci for C-reactive protein levels. Circulation. 2011;123(7):731-8.

8. Ho JE, Chen WY, Chen MH, Larson MG, McCabe EL, Cheng S, et al. Common genetic variation at the IL1RL1 locus regulates IL-33/ST2 signaling. J Clin Invest. 2013:123(10):4208-18.

9. de Vries PS, Chasman DI, Sabater-Lleal M, Chen M-H, Huffman JE, Steri M, et al. A meta-analysis of 120246 individuals identifies 18 new loci for fibrinogen concentration. Hum Mol Genet. 2016;25(2):358-70.

10. Naitza S, Porcu E, Steri M, Taub DD, Mulas A, Xiao X, et al. A genome-wide association scan on the levels of markers of inflammation in Sardinians reveals associations that underpin its complex regulation. PLoS Genet. 2012; 8(1):e1002480.

11. Durda P, Sabourin J, Lange EM, Nalls MA, Mychaleckyj JC, Jenny NS, et al. Plasma levels of soluble interleukin-2 receptor alpha: associations with clinical cardiovascular events and genome-wide association scan. Arterioscler Thromb Vasc Biol. 2015;35(10):2246-53.

12. Matteini AM, Li J, Lange EM, Tanaka T, Lange LA, Tracy RP, et al. Novel gene variants predict serum levels of the cytokines IL-18 and IL-1ra in older adults. Cytokine. 2014;65(1):10-6.

13. Tekola Ayele F, Doumatey A, Huang H, Zhou J, Charles B, Erdos M, et al. Genome-wide associated loci influencing interleukin (IL)-10, IL-1Ra, and IL-6 levels in African Americans. Immunogenetics. 2012;64(5):351-9.

14. Huang J, Sabater-Lleal M, Asselbergs FW, Tregouet D, Shin SY, Ding J, et al. Genome-wide association study for circulating levels of PAl-1 provides novel insights into its regulation. Blood. 2012;120(24):4873-81.

15. Levin AM, Mathias RA, Huang L, Roth LA, Daley D, Myers RA, et al. A metaanalysis of genome-wide association studies for serum total lgE in diverse study populations. J Allergy Clin Immunol. 2013;131(4):1176-84.

16. Viktorin A, Frankowiack M, Padyukov L, Chang Z, Melén E, Sääf A, et al. IgA measurements in over 12000 Swedish twins reveal sex differential. heritability and regulatory locus near CD30L. Hum Mol Genet. 2014;23(15): 4177-84.

17. Yang M, Wu Y, Lu Y, Liu C, Sun J, Liao M, et al. Genome-wide scan identifies variant in TNFSF13 associated with serum IgM in a healthy Chinese male population. PloS One. 2012;7(10):e47990-e.

18. Liao M, Ye F, Zhang B, Huang L, Xiao Q, Qin M, et al. Genome-wide association study identifies common variants at TNFRSF13B associated with IgG level in a healthy Chinese male population. Genes Immun. 2012;13(6): 509-13.

19. He M, Cornelis MC, Kraft P, van Dam RM, Sun Q, Laurie CC, et al. Genomewide association study identifies variants at the IL18-BCO2 locus associated with interleukin-18 levels. Arterioscler Thromb Vasc Biol. 2010;30(4):885-90.

20. Voruganti VS, Laston S, Haack K, Mehta NR, Smith CW, Cole SA, et al, Genome-wide association replicates the association of Duffy antigen receptor for chemokines (DARC) polymorphisms with serum monocyte chemoattractant protein-1 (MCP-1) levels in Hispanic children. Cytokine. 2012;60(3):634-8

21. Kwan JS, Hsu YH, Cheung CL, Dupuis J, Saint-Pierre A, Eriksson J, et al. Meta-analysis of genome-wide association studies identifies two loci associated with circulating osteoprotegerin levels. Hum Mol Genet. 2014;23(24):6684-93.

22. Huang J, Huffman JE, Yamakuchi M, Trompet S, Asselbergs FW, Sabater-Lleal $M$, et al. Genome-wide association study for circulating tissue plasminogen activator levels and functional follow-up implicates endothelial STXBP5 and STX2. Arterioscler Thromb Vasc Biol. 2014;34(5):1093-101

23. Choi SH, Ruggiero D, Sorice R, Song C, Nutile T, Vernon Smith A, et al. Six novel loci associated with circulating VEGF levels identified by a metaanalysis of genome-wide association studies. PLoS Genet. 2016;12(2): e1005874.
24. Yang X, Sun J, Gao Y, Tan A, Zhang H, Hu Y, et al. Genome-wide association study for serum complement C3 and C4 levels in healthy Chinese subjects. PLoS Genet. 2012;8(9):e1002916-e.

25. Smith NL, Huffman JE, Strachan DP, Huang J, Dehghan A, Trompet S, et al. Genetic predictors of fibrin D-dimer levels in healthy adults. Circulation. 2011;123(17):1864-72.

26. Yao C, Chen G, Song C, Keefe J, Mendelson M, Huan T, et al. Genome-wide mapping of plasma protein QTLs identifies putatively causal genes and pathways for cardiovascular disease. Nat Commun. 2018;9(1):3268.

27. Folkersen L, Fauman E, Sabater-Lleal M, Strawbridge RJ, Frånberg M, Sennblad B, et al. Mapping of 79 loci for 83 plasma protein biomarkers in cardiovascular disease. PLoS Genet. 2017;13(4):e1006706.

28. Barreiro LB, Tailleux L, Pai AA, Gicquel B, Marioni JC, Gilad Y. Deciphering the genetic architecture of variation in the immune response to mycobacterium tuberculosis infection. Proc Natl Acad Sci U S A. 2012;109(4): 1204-9.

29. Suhre K, Arnold M, Bhagwat AM, Cotton RJ, Engelke R, Raffler J, et al. Connecting genetic risk to disease end points through the human blood plasma proteome. Nat Commun. 2017;8:14357.

30. Sun BB, Maranville JC, Peters JE, Stacey D, Staley JR, Blackshaw J, et al. Genomic atlas of the human plasma proteome. Nature. 2018:558(7708):73-9.

31. Emilsson V, Ilkov M, Lamb JR, Finkel N, Gudmundsson EF, Pitts R, et al. Coregulatory networks of human serum proteins link genetics to disease. Science (New York). 2018;361 (6404):769-73.

32. Enroth S, Maturi V, Berggrund M, Enroth SB, Moustakas A, Johansson A, et al. Systemic and specific effects of antihypertensive and lipid-lowering medication on plasma protein biomarkers for cardiovascular diseases. Sci Rep. 2018;8(1):5531.

33. Deming Y, Xia J, Cai Y, Lord J, Del-Aguila JL, Fernandez MV, et al. Genetic studies of plasma analytes identify novel potential biomarkers for several complex traits. Sci Rep. 2016;6:18092.

34. Di Narzo AF, Telesco SE, Brodmerkel C, Argmann C, Peters LA, Li K, et al. Highthroughput characterization of blood serum proteomics of IBD patients with respect to aging and genetic factors. PLoS Genet. 2017;13(1):e1006565.

35. Sun W, Kechris K, Jacobson S, Drummond MB, Hawkins GA, Yang J, et al. Common Genetic Polymorphisms Influence Blood Biomarker Measurements in COPD. PLoS Genet. 2016;12(8):e1006011-e.

36. Hoglund J, Rafati N, Rask-Andersen M, Enroth S, Karlsson T, Ek WE, et al. Improved power and precision with whole genome sequencing data in genome-wide association studies of inflammatory biomarkers. Sci Rep. 2019; 9(1):16844.

37. Ligthart S, Marzi C, Aslibekyan S, Mendelson MM, Conneely KN, Tanaka T, et al. DNA methylation signatures of chronic low-grade inflammation are associated with complex diseases. Genome Biol. 2016;17(1):255.

38. Liang L, Willis-Owen SAG, Laprise C, Wong KCC, Davies GA, Hudson TJ, et al. An epigenome-wide association study of total serum immunoglobulin $\mathrm{E}$ concentration. Nature. 2015:520(7549):670-4.

39. Verschoor CP, McEwen LM, Kobor MS, Loeb MB, Bowdish DME. DNA methylation patterns are related to co-morbidity status and circulating Creactive protein levels in the nursing home elderly. Exp Gerontol. 2018;105: 47-52.

40. Verschoor CP, McEwen LM, Kohli V, Wolfson C, Bowdish DM, Raina P, et al. The relation between DNA methylation patterns and serum cytokine levels in community-dwelling adults: a preliminary study. BMC Genet. 2017;18(1):57.

41. Marzi C, Holdt LM, Fiorito G, Tsai PC, Kretschmer A, Wahl S, et al. Epigenetic signatures at $\mathrm{AQP3}$ and SOCS3 engage in low-grade inflammation across different tissues. PLoS One. 2016;11(11):e0166015.

42. Sun YV, Lazarus A, Smith JA, Chuang YH, Zhao W, Turner ST, et al. Genespecific DNA methylation association with serum levels of C-reactive protein in African Americans. PLoS One. 2013;8(8):e73480.

43. Zaghlool SB, Kühnel B, Elhadad MA, Kader S, Halama A, Thareja G, et al. Epigenetics meets proteomics in an epigenome-wide association study with circulating blood plasma protein traits. Nat Commun. 2020;11(1):15.

44. Ahsan M, Ek WE, Rask-Andersen M, Karlsson T, Lind-Thomsen A, Enroth S, et al. The relative contribution of DNA methylation and genetic variants on protein biomarkers for human diseases. PLoS Genet. 2017;13(9):e1 007005.

45. Flanagan JM. Epigenome-wide association studies (EWAS): past, present, and future. Methods Mol Biol (Clifton). 2015;1238:51-63.

46. Korte A, Farlow A. The advantages and limitations of trait analysis with GWAS: a review. Plant Methods. 2013;9:29. 
47. van Iterson M, van Zwet EW, Heijmans BT, the BC. Controlling bias and inflation in epigenome- and transcriptome-wide association studies using the empirical null distribution. Genome Biol. 2017;18(1):19.

48. Gagnon-Bartsch JA, Speed TP. Using control genes to correct for unwanted variation in microarray data. Biostatistics (Oxford). 2012;13(3):539-52.

49. Leek JT, Storey JD. Capturing heterogeneity in gene expression studies by surrogate variable analysis. PLoS Genet. 2007;3(9):1724-35.

50. Korte A, Vilhjálmsson BJ, Segura V, Platt A, Long Q, Nordborg M. A mixedmodel approach for genome-wide association studies of correlated traits in structured populations. Nat Genet. 2012;44(9):1066-71.

51. Rahmani E, Zaitlen N, Baran Y, Eng C, Hu D, Galanter J, et al. Sparse PCA corrects for cell type heterogeneity in epigenome-wide association studies. Nat Methods. 2016;13:443.

52. Zou J, Lippert C, Heckerman D, Aryee M, Listgarten J. Epigenome-wide association studies without the need for cell-type composition. Nat Methods. 2014;11:309.

53. Houseman EA, Molitor J, Marsit CJ. Reference-free cell mixture adjustments in analysis of DNA methylation data. Bioinformatics (Oxford). 2014;30(10):1431-9.

54. Trejo Banos D, McCartney DL, Patxot M, Anchieri L, Battram T, Christiansen $C$, et al. Bayesian reassessment of the epigenetic architecture of complex traits. Nature communications. 2020;11(1):2865.

55. Li Y, Willer CJ, Ding J, Scheet P, Abecasis GR. MaCH: using sequence and genotype data to estimate haplotypes and unobserved genotypes. Genet Epidemiol. 2010;34(8):816-34

56. Li Y, Willer C, Sanna S, Abecasis G. Genotype imputation. Annu Rev Genomics Hum Genet. 2009;10:387-406.

57. Zhang F, Chen W, Zhu Z, Zhang Q, Nabais MF, Qi T, et al. OSCA: a tool for omic-data-based complex trait analysis. Genome Biol. 2019;20(1):107.

58. Hillary RF, McCartney DL, Harris SE, Stevenson AJ, Seeboth A, Zhang Q, et al. Genome and epigenome wide studies of neurological protein biomarkers in the Lothian Birth Cohort 1936. Nat Commun. 2019;10(1):3160.

59. Taylor AM, Pattie A, Deary IJ. Cohort Profile Update: The Lothian Birth Cohorts of 1921 and 1936. Int J Epidemiol. 2018;47(4):1042-r.

60. Deary IJ, Gow AJ, Taylor MD, Corley J, Brett C, Wilson V, et al. The Lothian Birth Cohort 1936: a study to examine influences on cognitive ageing from age 11 to age 70 and beyond. BMC Geriatr. 2007;7:28.

61. Houseman EA, Accomando WP, Koestler DC, Christensen BC, Marsit CJ, Nelson $\mathrm{HH}$, et al. DNA methylation arrays as surrogate measures of cell mixture distribution. BMC bioinformatics. 2012;13(1):86.

62. Saffari A, Silver MJ, Zavattari P, Moi L, Columbano A, Meaburn EL, et al Estimation of a significance threshold for epigenome-wide association studies. Genet Epidemiol. 2018;42(1):20-33.

63. Wang K, Li M, Hakonarson H. ANNOVAR: functional annotation of genetic variants from high-throughput sequencing data. Nucleic Acids Res. 2010; 38(16):e164

64. Watanabe K, Taskesen E, van Bochoven A, Posthuma D. Functional mapping and annotation of genetic associations with FUMA. Nat Commun. 2017;8(1): 1826

65. Hansen KD. IlluminaHumanMethylation450kanno.ilmn12.hg19: Annotation for Illumina's 450k methylation arrays. R package version 060; 2016.

66. Võsa U, Claringbould A, Westra H-J, Bonder MJ, Deelen P, Zeng B, et al. Unraveling the polygenic architecture of complex traits using blood eQTL metaanalysis. bioRxiv. 2018; https://doi.org/10.1101/447367:447367.

67. Giambartolomei C, Vukcevic D, Schadt EE, Franke L, Hingorani AD, Wallace $C$, et al. Bayesian test for colocalisation between pairs of genetic association studies using summary statistics. PLoS Genet. 2014;10(5):e1004383.

68. Guo H, Fortune MD, Burren OS, Schofield E, Todd JA, Wallace C. Integration of disease association and $\mathrm{eQTL}$ data using a Bayesian colocalisation approach highlights six candidate causal genes in immune-mediated diseases. Hum Mol Genet. 2015;24(12):3305-13.

69. Durinck S, Spellman PT, Birney E, Huber W. Mapping identifiers for the integration of genomic datasets with the R/bioconductor package biomaRt. Nat Protoc. 2009;4(8):1184.

70. Durinck S, Moreau Y, Kasprzyk A, Davis S, De Moor B, Brazma A, et al. BioMart and Bioconductor: a powerful link between biological databases and microarray data analysis. Bioinformatics (Oxford). 2005;21(16):3439-40.

71. Liberzon A, Birger C, Thorvaldsdottir H, Ghandi M, Mesirov JP, Tamayo P. The Molecular Signatures Database (MSigDB) hallmark gene set collection. Cell Syst. 2015;1(6):417-25.

72. Tenenbaum D. KEGGREST: client-side REST access to KEGG. R package version; 2016. p. 1
73. MacArthur J, Bowler E, Cerezo M, Gil L, Hall P, Hastings E, et al. The new NHGRI-EBI Catalog of published genome-wide association studies (GWAS Catalog). Nucleic Acids Res. 2017;45(D1):D896-901.

74. Liu JZ, van Sommeren S, Huang H, Ng SC, Alberts R, Takahashi A, et al. Association analyses identify 38 susceptibility loci for inflammatory bowel disease and highlight shared genetic risk across populations. Nat Genet. 2015;47(9):979-86.

75. Marioni RE, Harris SE, Zhang Q, MCRae AF, Hagenaars SP, Hill WD, et al. GWAS on family history of Alzheimer's disease. Transl Psychiatry. 2018;8(1):99.

76. Hemani G, Zheng J, Elsworth B, Wade KH, Haberland V, Baird D, et al. The MR-base platform supports systematic causal inference across the human phenome. eLife. 2018;7:e34408.

77. He X, Fuller Chris K, Song Y, Meng Q, Zhang B, Yang X, et al. Sherlock: detecting gene-disease associations by matching patterns of expression QTL and GWAS. Am J Hum Genet. 2013;92(5):667-80.

78. Bonder MJ, Luijk R, Zhernakova DV, Moed M, Deelen P, Vermaat M, et al. Disease variants alter transcription factor levels and methylation of their binding sites. Nat Genet. 2017:49(1):131-8.

79. Morgan AR, Touchard S, Leckey C, O'Hagan C, Nevado-Holgado AJ, Barkhof $F$, et al. Inflammatory biomarkers in Alzheimer's disease plasma. Alzheimers Dement. 2019;15(6):776-87.

80. Cao W, Zheng H. Peripheral immune system in aging and Alzheimer's disease. Mol Neurodegener. 2018;13(1):51.

81. Westra HJ, Peters MJ, Esko T, Yaghootkar H, Schurmann C, Kettunen J, et al. Systematic identification of trans eQTLs as putative drivers of known disease associations. Nat Genet. 2013;45(10):1238-43.

82. Schnabel RB, Baumert J, Barbalic M, Dupuis J, Ellinor PT, Durda P, et al. Duffy antigen receptor for chemokines (Darc) polymorphism regulates circulating concentrations of monocyte chemoattractant protein-1 and other inflammatory mediators. Blood. 2010;115(26):5289-99.

83. Rot A. Contribution of Duffy antigen to chemokine function. Cytokine Growth Factor Rev. 2005;16(6):687-94.

84. Tsaprouni LG, Yang T-P, Bell J, Dick KJ, Kanoni S, Nisbet J, et al. Cigarette smoking reduces DNA methylation levels at multiple genomic loci but the effect is partially reversible upon cessation. Epigenetics. 2014;9(10):1382-96.

85. Joehanes R, Just AC, Marioni RE, Pilling LC, Reynolds LM, Mandaviya PR, et al. Epigenetic signatures of cigarette smoking. Circ Cardiovasc Genet. 2016;9(5):436-47.

86. Zeilinger S, Kühnel B, Klopp N, Baurecht H, Kleinschmidt A, Gieger C, et al. Tobacco smoking leads to extensive genome-wide changes in DNA methylation. PloS One. 2013;8(5):e63812-e.

87. Zhang Y, Breitling LP, Balavarca Y, Holleczek B, Schottker B, Brenner $H$. Comparison and combination of blood DNA methylation at smokingassociated genes and at lung cancer-related genes in prediction of lung cancer mortality. Int J Cancer. 2016;139(11):2482-92.

88. Elliott HR, Tillin T, McArdle WL, Ho K, Duggirala A, Frayling TM, et al. Differences in smoking associated DNA methylation patterns in South Asians and Europeans. Clin Epigenet. 2014;6(1):4.

89. Philibert RA, Beach SRH, Brody GH. Demethylation of the aryl hydrocarbon receptor repressor as a biomarker for nascent smokers. Epigenetics. 2012; 7(11):1331-8.

90. Dogan MV, Shields B, Cutrona C, Gao L, Gibbons FX, Simons R, et al. The effect of smoking on DNA methylation of peripheral blood mononuclear cells from African American women. BMC Genomics. 2014;15:151.

91. Kodal JB, Kobylecki CJ, Vedel-Krogh S, Nordestgaard BG, Bojesen SE. AHRR hypomethylation, lung function, lung function decline and respiratory symptoms. The European respiratory journal. 2018;51(3):1701512.

92. Shiels MS, Katki HA, Freedman ND, Purdue MP, Wentzensen N, Trabert B, et al. Cigarette smoking and variations in systemic immune and inflammation markers. J Natl Cancer Inst. 2014:106(11):dju294.

93. Fernandez-Egea E, Scoriels L, Theegala S, Giro M, Ozanne SE, Burling K, et al. Cannabis use is associated with increased CCL11 plasma levels in young healthy volunteers. Prog Neuro-Psychopharmacol Biol Psychiatry. 2013;46:25-8.

94. Krisiukeniene A, Babusyte A, Stravinskaite K, Lotvall J, Sakalauskas R, Sitkauskiene B. Smoking affects eotaxin levels in asthma patients. J Asthma. 2009;46(5):470-6.

95. Davis BK, Roberts RA, Huang MT, Willingham SB, Conti BJ, Brickey WJ, et al. Cutting edge: NLRC5-dependent activation of the inflammasome. Journal Immunol. 2011;186(3):1333-7.

96. Ma C, Wu W, Lin R, Ge Y, Zhang C, Sun S, et al. Critical role of CD6highCD4+ T cells in driving Th1/Th17 cell immune responses and mucosal inflammation in IBD. J Crohns Colitis. 2019;13(4):510-24. 
97. Ren Y, Jiao X, Zhang L. Expression level of fibroblast growth factor 5 (FGF5) in the peripheral blood of primary hypertension and its clinical significance. Saudi J Biol Sci. 2018;25(3):469-73.

98. Sandborn WJ, Feagan BG, Fedorak RN, Scherl E, Fleisher MR, Katz S, et al. A randomized trial of Ustekinumab, a human interleukin-12/23 monoclonal antibody, in patients with moderate-to-severe Crohn's disease. Gastroenterology. 2008;135(4):1130-41.

99. Williams MA, O'Callaghan A, Corr SC. IL-33 and IL-18 in inflammatory bowel disease etiology and microbial interactions. Front Immunol. 2019;10:1091.

100. Borish LC, Steinke JW. 2. Cytokines and chemokines. J Allergy Clin Immunol. 2003;111(2 Suppl):S460-75.

101. Hillary RF, Trejo-Banos D, Kousathanas A, McCartney DL, Harris SE, Stevenson AJ, et al. Linear Regression GWAS Proteins. Edinburgh Datashare. 2020; https://doi.org/10.7488/ds/2814.

102. Hillary RF, Trejo-Banos D, Kousathanas A, McCartney DL, Harris SE, Stevenson AJ, et al. BayesR+ GWAS Proteins. Edinburgh Datashare. 2020; https://doi. org/10.7488/ds/2854.

103. Hillary RF, Trejo-Banos D, Kousathanas A, McCartney DL, Harris SE, Stevenson AJ, et al. Linear Regression EWAS Proteins. Edinburgh Datashare. 2020; https://doi.org/10.7488/ds/2818.

104. Hillary RF, Trejo-Banos D, Kousathanas A, McCartney DL, Harris SE, Stevenson AJ, et al. OSCA EWAS Proteins. Edinburgh Datashare. 2020; https://doi.org/ $10.7488 / \mathrm{ds} / 2817$

105. Hillary RF, Trejo-Banos D, Kousathanas A, McCartney DL, Harris SE, Stevenson AJ, et al. BayesR+ EWAS Proteins. Edinburgh Datashare. 2020; https://doi. org/10.7488/ds/2816

106. Hillary RF, Trejo-Banos D, Kousathanas A, McCartney DL, Harris SE, Stevenson AJ, et al. GWAS Summary Statistics on 70 Inflammatory Proteins - OLS Regression GWAS. 2020. https://www.ebi.ac.uk/gwas/:GCST90000437GCST90000506.

\section{Publisher's Note}

Springer Nature remains neutral with regard to jurisdictional claims in published maps and institutional affiliations.

Ready to submit your research? Choose BMC and benefit from:

- fast, convenient online submission

- thorough peer review by experienced researchers in your field

- rapid publication on acceptance

- support for research data, including large and complex data types

- gold Open Access which fosters wider collaboration and increased citations

- maximum visibility for your research: over $100 \mathrm{M}$ website views per year

At $\mathrm{BMC}$, research is always in progress.

Learn more biomedcentral.com/submissions 